\title{
Chinese International Student Sexual Harassment on U.S. College Campuses
}

\author{
Yunling Chang \\ Sakina Ali \\ Ankita Sahu \\ Sidai Dong \\ Carly W. Thornhill \\ Polet Milian \\ Linda G. Castillo \\ Texas A\&M University, USA
}

\begin{abstract}
The \#MeToo movement has brought the issue of sexual harassment to U.S. college campuses. Most scholarly work in this area focuses on White American women with little information on international student experiences. Because sexual harassment is considered hush-hush (shi) and taboo, many Chinese international students may not question harassment behaviors they experience. For many Chinese women attending a U.S. university, their first public discussion may occur during student orientation. Thus, students come to college campuses with varying levels of awareness of sexual harassment. Given the growing number of Chinese international students, the purpose of this article is to provide an overview of their experiences and perceptions of sexual harassment. The article also provides recommendations for university personnel working with international students.
\end{abstract}

Keywords: higher education, international students, sexual harassment

\section{INTRODUCTION}

The "\#MeToo" movement has brought the topic of sexual harassment back to the forefront particularly on college campuses in the United States. Research on sexual harassment in higher education points to the negative impact on mental 
health and educational outcomes (National Academies of Sciences, Engineering, and Medicine, 2018). Although there is growing research in this area, little is known how the experience of sexual harassment affects international students, particularly Chinese women, who represent about a third of international students studying at U.S. college campuses (Ho et al., 2018).

For many international students, experiencing sexual harassment can be a triple risk for their well-being (Poyrazli, 2015). Unfamiliarity with university policies and procedures, fear that filing a complaint will endanger their student status, and shame that family members will find out about the incident may prevent students from seeking assistance from campus resources (Swanbrow Becker et al., 2018).

As discussing sexual matters is considered taboo in Chinese culture and often not discussed with friends or family (Leiber et al., 2009), international students may lack knowledge of sex-related topics like sexual harassment. As such, Chinese international students have limited knowledge of what constitutes sexually harassing behaviors. Their first introduction to the concept often occurs at student orientation. Given the lack of scholarly work in this area, the overall purpose of this paper is to (1) provide an overview of Chinese international students' experiences and perceptions of sexual harassment, (2) introduce how sexual harassment is conceptualized, and (3) discuss interventions when working with Chinese international students.

\section{LITERATURE REVIEW}

\section{Experiences and Perceptions of Sexual Harassment}

Tang and colleagues (1996) were the first to examine sexual harassment of Chinese women. In a study of 849 Chinese college student participants, approximately one in four women experienced some form of sexual harassment. The study also found that commonly identified sexual harassment behaviors, such as unwanted pressure for dates and unwanted disclosure of personal matters, were not perceived as sexual harassment by $80 \%$ of the participants. More recently, studies found that the most common type of sexual harassment behavior on China's college campuses includes sexually suggestive comments, inappropriate physical contact, leering or making sexual gestures, commenting on one's appearance sexually, and persistent invitations to go out on dates regardless of rejection (Chan et al., 2019).

Studies suggest that among women of diverse racial and ethnic backgrounds, Asian/Pacific Islander women were the least likely to report sexual and physical victimization (Ho et al., 2018). Sexual harassment as a hush-hush (shi) topic speaks to the secretive nature of the issue and explains why many avoid reporting it (Leiber et al., 2009). Other Asian cultural factors like saving face, avoidance of shame, and filial piety may impact their perceptions of sexual harassment as well as whether such incidents are reported (Chan, 2012). For instance, keeping a family's reputation is of utmost importance to Chinese international students and 
to acknowledge that one has experienced sexual harassment by filing a complaint may be viewed as bringing shame to the family (Ho et al., 2018).

\section{Conceptualization of Sexual Harassment}

Most sexual harassment research is based on Fitzgerald and colleagues' (1995) sexual harassment model. The Fitzgerald et al.'s model conceptualizes sexual harassment as a multidimensional construct that consists of (1) unwanted sexual attention, (2) gender harassment, and (3) sexual coercion. Unwanted sexual attention includes verbal and nonverbal behaviors that are sexual in nature and are unreciprocated by the other party. Gender harassment involves both verbal and nonverbal behaviors that are designed to denigrate women, such as sexist and misogynistic comments or behaviors. Sexual coercion, or quid pro quo, is the expectation that engaging in a sexual behavior will result in a work-related benefit for the party that holds less power.

Because Fitzgerald et al.'s (1995) model was based on a Western concept of the construct, Tang et al. (1996) developed a model of how people from China conceptualized sexual harassment. At a university in Hong Kong, Tang and colleagues (1996) administered a questionnaire to gather information about awareness, experiences, and reactions to sexual harassment. Study findings revealed similarities with the Western conceptualization of sexual harassment. Similar to Fitzgerald et al.'s (1995) model, unwanted intimate behaviors (i.e., unwanted sexual attention) were classified as sexual harassment by participants. The unwanted intimate behaviors consisted of coercion for sexual activity, caressing legs, touching of sex organs, and unwanted sexual phone calls.

Conversely, gender harassment was not considered sexual harassment to participants in the Tang et al. studies (1996). Gender harassment consisted of sexist remarks and misogynistic behaviors. This differs from White American female college students who identify sexist remarks as a form of sexual harassment (Kalof et al, 2001).

Along with gender harassment, Chinese student participants did not consider covert seductive behaviors (i.e., sexual coercion) as sexual harassment (Tang et al., 1996). Unwanted pressure for dates and unwanted disclosure of personal or emotional matters constitute covert seductive behaviors. However, quid pro quo behaviors, such as demanding sexual contact in exchange for certain favors or benefits, were considered sexual harassment by Chinese student participants.

Based on Tang and colleagues (1996) it appears that overt behaviors, such as unwanted touching, and quid pro quo actions are more readily acknowledged as sexual harassment. However, covert behaviors were not perceived as sexual harassment. Tang and colleagues (1996) speculated that given Chinese students' limited education about sexual harassment may have contributed to this finding. 


\section{INTERVENTIONS WITH CHINESE INTERNATIONAL STUDENTS}

When working with Chinese international students who have experienced sexual harassment, universities can intervene on multiple levels to make sure their needs are addressed. For university staff at counseling centers, acknowledgment of Chinese cultural values such as filial piety, shame, and saving face should be taken into account when working with Chinese international students (Ho et al., 2018). Particularly for Chinese women, it is important for university staff to incorporate a social justice and advocacy perspective within counseling (Morrow \& Hawxhurst, 1998).

Given Tang et al.'s (1996) study that Chinese students do not view sexist remarks or covert seductive behaviors as sexual harassment, university staff can take a preventative approach by educating students on dating norms in the United States and the types of behaviors that are not appropriate. By providing Chinese international students with information about different types of sexual harassment, students can make sense of their experience when such behaviors are encountered since many may not have realized they had been sexually harassed.

Research suggests that adapting Acceptance and Commitment Therapy (ACT) to fit Chinese cultural values can be helpful for Chinese international students seeking help for coping with stress (Xu et al., 2020). ACT draws upon Eastern philosophies and Buddhist principles to create a treatment that accepts pain as a part of life, which fits well with Chinese international students' cultural values. University counseling center staff can provide ACT by integrating Chinese cultural references and metaphors, using a group therapy format, and providing self-disclosure about their own acculturative experiences $(\mathrm{Xu}$ et al., 2020). As noted by $\mathrm{Xu}$ and colleagues, these adaptations can help students to connect with their university counselor on a personal level as well as alleviate the sense of shame associated with seeking mental health treatment.

Student affairs professionals can also position themselves to provide interventions when working with Chinese international students. University leaders must work with on-campus multicultural centers and international student services to provide culturally informed training to students and administrators. For instance, Columbia and Barnard University's student affairs leaders recently implemented the Sexual Health Initiative to Foster Transformation (SHIFT) program. This program aims to advancing research on the social and cultural roots of sexual assault on a college campus and is supported with a budget and administrator training. Trainings are also provided to student leaders and athletes on consent in Asian cultures (Hirsch et al., 2018).

Fraser International College at the University in Vancouver, British Columbia provides another example of prevention programming for international students (Reid \& Dunlap, 2017). The university incorporated sexual and sexual assault information into all orientations, curricula, and program for new international students. Students are required to take a semester-long class that covers consent and healthy communication, sexual health and gender orientation, and cultural and societal norms around sex. The program also creates a safe environment for students by allowing anonymous submission of questions. 
In sum, campus implementation of new initiatives requires the support of campus leaders, student affairs divisions, and counseling centers. This means taking intentional measures to implement university-wide programs and policies that support Chinese international students. Furthermore, Chinese international students who have been sexually harassed can benefit from mental health treatment that has been adapted to incorporate cultural values and alleviate shame that may occur for this population. Campuses that promote inclusion and student safety must incorporate culturally competent efforts that make the international student community feel safe in a place that is so far from home.

\section{REFERENCES}

Chan, J. K. S., Lam, K. K. Y., Cheung, C. C. M., \& Lo, J. T. Y. (2019). Break the silence: Territory-wide study on sexual harassment of university students in Hong Kong. Equal Opportunities Commission.

Chan, K. L. (2012). The role of Chinese face in the perpetration of dating partner violence. Journal of Interpersonal Violence, 27(4), 793-811. https:// doi.org/10.1177/0886260511423242

Fitzgerald, L. F., Gelfand, M. J., \& Drasgow, F. (1995). Measuring sexual harassment: Theoretical and psychometric advances. Basic and Applied Social Psychology, 17, 425-445. https://doi.org/10.1207/s15324834 basp1704_2

Hirsch, J. S., Reardon, L., Khan, S., Santelli, J. S., Wilson, P. A., Gilbert, L., Wall, M. M., \& Mellins, C. (2018). Transforming the campus climate: Advancing mixed-methods research on the social and cultural roots of sexual assault on a college campus. Voices, 13(1), 23-54. https://doi.org/10.1111/voic.12003

Ho, I. K., Ding, K. T., Bellefontaine, S. M., \& Irving, A. L. (2018). Cultural adaptation and sexual harassment in the lives of Asian American women. Women \& Therapy, 41, 281-297. https://doi.org/10.1080/02703149.2018. 1430300

Kalof, L., Eby, K. K., Matheson, J. L., \& Kroska, R. J. (2001). The influence of race and gender on student self-reports of sexual harassment by college professors. Gender \& Society, 15(2), 283-302. https://doi.org/10.1177/ 089124301015002007

Leiber, E., Chin, D., Li, L., Rotheram-Borus, M. J., Detels, R., Wu, Z., \& Guan, J. (2009). Sociocultural contexts and communication about sex in China: Informing HIV/STD prevention programs. Aids Education Prevention, 21(5), 415-429. https://doi.org/10.1521/aeap.2009.21.5.415

Morrow, S. L., \& Hawxhurst, D. M. (1998). Feminist therapy: Integrating political analysis in counseling and psychotherapy. Women \& Therapy, 21(2), 37-50. https://doi.org/10.1300/J015v21n02_03

National Academies of Sciences, Engineering, and Medicine (2018). Sexual harassment of women: Climate, culture, and consequences in academic sciences, engineering, and medicine. The National Academies Press. https://doi.org/10.17226/24994 
Poyrazli, S. (2015). Psychological symptoms and concerns experienced by international students: Outreach implications for counseling centers. Journal of International Students, 5, 306-312. https://doi.org/10.32674/jis.v5i3.424

Reid, J., \& Dunlap, J. (2017, November 15). Sex and the International Student. Inside Higher Education. https://www.insidehighered.com/views/2017/11/ 15/international-students-need-different-education-programs-sexual-assaultand-other

Swanbrow Becker, M. A., Dong, S., Kronholz, J., \& Brownson, C. (2018). Relationships between stress and psychosocial factors with sources of helpseeking among international students. Journal of International Students, 8, 1636-1661. https://doi.org/10.32674/jis.v8i4.222

Tang, C. S. K., Yik, M. S., Cheung, F. M., Choi, P. K., \& Au, K. C. (1996). Sexual harassment of Chinese college students. Archives of Sexual Behavior, 25(2), 201-215. https://doi.org/10.1007/bf02437936

Xu, H., Obrien, W. H., \& Chen, Y. (2020). Chinese international student stress and coping: A pilot study of acceptance and commitment therapy. Journal of Contextual Behavioral Science, 15, 135-141. https://doi.org/10.1016/ j.jcbs.2019.12.010

YUNLING CHANG, MS, is a doctoral candidate in the Department of Educational Psychology at Texas A\&M University and a Psychology Intern at Texas A\&M Counseling \& Psychological Services. Her major research interests lie in the area of international student population and trauma (particularly sexual and racial). Email: yunl30@tamu.edu

SAKINA ALI, MEd, is a doctoral candidate in the Department of Educational Psychology at Texas A\&M University. Her major research interests include examining the impact of religion/spirituality, patriarchal ideologies, and acculturative stress on mental health outcomes for marginalized populationsspecifically South Asian Americans and LGBTQ+ people. Email: sakina.ali@tamu.edu

ANKITA SAHU, MEd. is a doctoral candidate in the Department of Educational Psychology at Texas A\&M University. Her research interests focus on multicultural counseling training and supervision. Email: asahu@tamu.edu

SIDAI DONG, MA, MEd, is a doctoral candidate in the Department of Educational Psychology at Texas A\&M University and a Psychology Intern at Texas A\&M Counseling \& Psychological Services. Her research interests focus on understanding the intersections among cognitive, behavioral, emotional development, and mental health outcomes for emerging adults, people with disabilities, and their caregivers. Email: sdong02@tamu.edu

CARLY W. THORNHILL, MEd, is a second-year doctoral student in the Department of Educational Psychology at Texas A\&M University. Her research interests focus on the areas of sexual assault, the attainment of higher education, and multiculturalism. Email: carlywthornhill@tamu.edu 
POLET MILIAN (she/her), MSEd, is a second-year doctoral student in the Department of Educational Psychology at Texas A\&M University. Her research interests focus broadly on multicultural psychology and specifically on the areas of Latinx persistence and mental health particularly among immigrant populations. Email: milianp@tamu.edu

LINDA G. CASTILLO, $\mathrm{PhD}$, is a professor in the Department of Educational Psychology at Texas A\&M University. Her expertise and research focus on Latina educational persistence and mental health and scale development of cultural constructs. Email: 1castillo@tamu.edu 